\title{
Screening for Intimate Partner Violence
}

\author{
Mary T. Paterno, CNM, RN, PhD and \\ An assistant professor at the University of Massachusetts Amherst in Amherst, MA and practices \\ nurse-midwifery at Baystate Medical Practices Pioneer Women's Health in Greenfield, \\ Massachusetts \\ Jessica E. Draughon, RN, PhD, SANE-A \\ An assistant professor at the Betty Irene Moore School of Nursing at UC Davis and is a certified \\ Sexual Assault Nurse Examiner
}

\section{Background}

Intimate partner violence (IPV) refers to emotional, physical, or sexual violence from a current or former intimate partner, including experience of coercion, stalking, and psychological aggression. ${ }^{1}$ Globally, $30 \%$ of women experience physical or sexual IPV in their lifetime, increasing to $50 \%$ of women when including emotional/psychological violence. ${ }^{2}$ Beyond immediate injuries, women exposed to IPV experience an array of poor health outcomes compared to non-abused women. ${ }^{2}$ IPV is associated with chronic pain, depression, anxiety, post-traumatic stress disorder, and substance use. ${ }^{2}$

Further, IPV is a serious concern during pregnancy. IPV during pregnancy is associated with many adverse outcomes including preterm birth, ${ }^{2,3}$ intrauterine growth restriction, ${ }^{2}$ low birthweight, ${ }^{2,3}$ and neonatal intensive care admission. ${ }^{3}$ Maternal health outcomes include increased risk for gestational hypertension, ${ }^{3}$ vaginal bleeding, ${ }^{3}$ urinary tract infections, ${ }^{3}$ severe nausea, ${ }^{3}$ vomiting or dehydration, ${ }^{3}$ and prenatal and postpartum depression. ${ }^{4}$ For some, pregnancy is protective against IPV; for others, IPV begins during pregnancy or increases in severity. ${ }^{4}$ Violence during pregnancy is associated with young age, separation/ divorce during pregnancy, substance use, financial difficulties, and unplanned pregnancy. ${ }^{2,4}$ This article reviews evidence-based best practice to implement routine screening for and response to IPV.

\section{Screening for Intimate Partner Violence}

Screening for IPV is effective when done in a therapeutic and systematic manner. The United States Preventive Services Taskforce (USPSTF) released updated recommendations for screening in 2012, ${ }^{5}$ concluding that screening can identify women experiencing IPV and screening has minimal adverse effects. The USPSTF joins various professional groups,

\footnotetext{
Corresponding author: Mary T. Paterno, CNM, RN, PhD, Assistant Professor, University of Massachusetts Amherst, College of Nursing, 651 N. Pleasant Street, Skinner Hall, Room 130, Amherst, MA 01003, (413) 545-1243, (413) 577-2550, mtpaterno@nursing.umass.edu, mary.t.paterno@gmail.com.

The authors have no conflicts of interest to disclose.
} 
including the American College of Nurse-Midwives, in recommending screening women of reproductive age for IPV and providing referrals. 5,6

\section{Barriers to Screening}

Although routine IPV screening is recommended, not all clinicians do so. A recent systematic review reported only $9 \%-40 \%$ of clinicians routinely screen for IPV. ${ }^{7}$ The literature documents several barriers to screening, including personal discomfort with the topic; inadequate resources; lack of time, training, or privacy to screen; perceptions that IPV screening is not the clinician's role; fear of offending patients; and not knowing how to respond to a woman disclosing IPV. ${ }^{8}$ Many of these barriers can be addressed through proper training and development of a systematic screening protocol. Certified nursemidwives and certified midwives, as primary care providers of women's health, play a crucial role in identifying and responding to IPV.

\section{Best Practices for Screening For Intimate Partner Violence}

\section{Be Systematic}

$\mathrm{O}^{\prime}$ Reilly et $\mathrm{al}^{9}$ identified practices that increase IPV identification, including using a systematic screening protocol and consistently using the same screening tool. The protocol should include: 1) who will conduct the screening, 2) the method of screening that will be used, 3) who will respond to a positive screen, and 4) how often to screen. The American College of Obstetricians and Gynecologists ${ }^{10}$ and other experts on IPV ${ }^{11}$ recommend every woman of reproductive age be screened upon entry to health care and that IPV screening be repeated at all well-visits and annual exams. In addition, they recommend pregnant women be screened once per trimester as well as at a postpartum visit. ${ }^{10}$ There is no clear consensus in the literature as to who should conduct the screen (ie, midwife versus other staff) or the best method for conducting screening (ie, on paper, verbally).

\section{Develop a Script}

By developing an IPV screening script in advance, midwives can practice screening with friends and colleagues in order to sound natural and engaging and reduce potential anxiety and discomfort. Starting with a normalizing statement legitimizes the need for a conversation about IPV and effectively communicates to the woman she is not alone in her experiences ${ }^{7,12}$ A sample script is presented in Table 1. Clinicians should avoid using potentially stigmatizing terms such as "battered", "abused", and "raped". ${ }^{10}$ Using behaviorally specific terms is preferred, such as hit, slapped, or kicked, versus "abused" or "domestic violence". ${ }^{1}$ A woman may not recognize herself as a "victim" of IPV and use of value-laden terms may decrease her ability to define experiences as abusive or violent, which can negatively impact her willingness to disclose IPV.

\section{Screening Instruments}

Using a valid and reliable screening tool is essential; there are many valid and reliable screening tools developed for use and tested in primary care or episodic care settings ${ }^{5}$ (Table 2). One commonly used tool is the Abuse Assessment Screen, ${ }^{13}$ which is included in Table 1. A "yes" response to any question indicates a positive IPV screen and completing the 
screening requires less than one minute when all responses are "no."13 Clinicians should avoid asking questions that guarantee a negative response such as "You're not abused are you?." In addition, asking a single question to screen for IPV is insufficient. A common screening question is "Do you feel safe at home?." While relevant, this question has low sensitivity for IPV ${ }^{5}$; there are many reasons a woman may not feel safe at home that are unrelated to IPV, for example neighborhood violence or unstable housing structures. A woman who feels unsafe at home may not be experiencing IPV, and a woman who is experiencing IPV may feel safe at home.

\section{Mandatory Reporting}

Prior to implementing routine IPV screening, clinicians should be aware of mandatory reporting laws in their practice state/jurisdiction. When practicing in a state that requires reporting of all IPV-related injuries, as opposed to only life-threatening or injuries involving a weapon, it is imperative to inform women of mandated reporting requirements before conducting the screening. This promotes and respects women's autonomy to decide whether to disclose IPV with sufficient information to make such a decision. ${ }^{12,14}$

\section{Privacy}

IPV screening should be conducted in private, one-on-one, and without children or other persons present. ${ }^{7} \mathrm{~A}$ woman accompanied by her abuser will most likely be unable to answer screening questions honestly. Women also may be unwilling to disclose IPV in front of family members or friends. ${ }^{7}$ Informing the woman and those accompanying her that it is clinic/hospital policy to always speak with patients alone creates opportunities for screening privately. Alternatively, screening in private could occur when escorting the woman to the bathroom. The need for privacy highlights the importance of using an official medical translator when language barriers are present, as opposed to bilingual family members. Trained medical translators keep conversations confidential and increase the fidelity of patient-care encounters.

\section{Therapeutic Communication}

Finally, therapeutic communication is an important component of IPV screening. Creating a lasting and trusting relationship with a woman is a major clinical goal. One relevant consideration is to meet a woman at eye-level versus standing over her, especially in the confines of small patient rooms. ${ }^{12}$ Invasion of personal space may be a trigger for anxiety and other post-traumatic stress symptoms, ${ }^{15}$ which could interfere with her willingness to disclose or her comfort in disclosing IPV. With the proliferation of computers or tablets in patient-care settings, it is easy and often expedient to direct attention toward an electronic device. Focusing on the computer detracts from the therapeutic relationship, ${ }^{12}$ and women are less likely to disclose IPV to a person whom they do not trust. ${ }^{7,16}$

\section{Responding to a Positive Screen for Intimate Partner Violence}

Women have diverse reasons for not disclosing IPV at any given interaction and her autonomy to make this decision should be respected. ${ }^{12}$ In the event of a positive screen, time will be necessary for the response. The midwife's time will be invested in working with the 
woman who does disclose IPV, tailoring the interaction to that individual in order to improve general health, maternal, and fetal outcomes.

\section{Immediate Response}

The first response is to thank the woman for answering, and to acknowledge the step she has taken in sharing her experiences, whether current or past. ${ }^{12}$ Consider a statement such as "Thank you for answering. I'm sorry to hear that this has happened/is happening to you. You deserve to be treated with respect and no one should be hurt by their partner." See Tables 1 and 3 for additional examples. It is important to avoid statements that could incite fear of her partner discovering she disclosed IPV. Making an immediate offer to call police is well intentioned but women have found this response to be unhelpful ${ }^{16}$; concerns about police involvement has been found to be a contributor to nondisclosure of IPV. ${ }^{14}$ Instead: 1) ask the woman if she wants help at this time, 2) offer assistance and choices, and 3) encourage safety planning without coercion. ${ }^{12}$ Subtle differences in language such as "may I share some resources with you" instead of "let me give you some resources" can help build trust. If a woman does not desire assistance after disclosure, a compassionate and non-judgmental response communicates that resources and help are available if she changes her mind. ${ }^{12}$ See Table 3 for example language.

\section{Next Steps}

Following a positive screen, the clinician has the responsibility to take the following additional actions: 1) complete additional assessments, 2) offer safety planning, and 3) offer referrals. ${ }^{11,17}$ Gathering detailed information through additional assessment to clarify the woman's experience and situation is necessary. Before completing additional assessments, remind the woman about confidentiality limits if proceeding might involve notifying legal authorities. Determining timing of the last abusive event and whether sexual violence was involved will direct provision of referrals to sexual assault/forensic nursing services, as the jurisdiction where the assault occurred will have evidence collection time limits which are usually $72-120$ hours. There is a strong correlation between IPV and depression,,${ }^{11}$ posttraumatic stress, ${ }^{11}$ substance use, ${ }^{11,17}$ traumatic brain injury, ${ }^{11}$ and reproductive coercion. ${ }^{10}$ Thus, screening for depression and substance abuse are also recommended. Because women often underestimate the level of danger in their relationship, ${ }^{18}$ the addition of a lethality assessment, such as the Danger Assessment, ${ }^{18}$ which identifies risk factors for intimate partner homicide in the next 12 months, would be prudent. This 20 -item self-report tool provides a lethality risk stratification score (variable, increased, severe, extreme), ${ }^{18}$ which may serve as a natural segue into discussing safety planning. Information about the Danger Assessment and other resources are listed in Table 4.

Many women are not ready to leave an abusive relationship at the time of screening. ${ }^{16}$ Strategies to enhance safety while remaining in the abusive relationship are critical, as is creating a plan to implement if and when she decides to leave, because attempting to leave an abuser without a safety plan can actually increase danger. ${ }^{16,18}$ Strategies may include keeping copies of vital records (eg, birth certificates, social security cards, passports) and money hidden or at a trusted friend's house for a quick escape, discussing ways to protect young children, and knowing/taking steps for obtaining a restraining order. This may seem 
an overwhelming amount of content to review for busy clinicians; if time is constrained the clinician can refer the woman to someone who provides safety planning, ${ }^{11,17}$ such as a community-based intimate partner violence advocate group or crisis hotline.

Referrals to local resources such as a social worker, advocacy group, domestic violence specialist, forensic nursing program, branch of the health department, or a shelter can be given. There may be limited local resources in rural areas but national resources, such as the National Domestic Violence Hotline (1-800-799-SAFE) and the National Sexual Assault Hotline (1-800-656-HOPE), are always available (Table 3). Best practice includes offering to make an electronic or telephonic connection to resources with the woman at the time of screening, ${ }^{11,12}$ which could involve offering to make a call on her behalf and/or sitting with her during a phone referral. It is not a clinical failure if a woman does not want help or seem receptive to receiving assistance. In this situation, the goal is to communicate with a warm, non-judgmental response that the woman is in a safe place in which to discuss and receive assistance related to IPV when she is ready. ${ }^{12}$

\section{Conclusion}

All clinicians should routinely screen women for IPV. The development of a systematic screening protocol for use in clinical practice, which includes a plan for immediate response and action items when a woman discloses IPV, will assist in providing evidence-based screening. Prior to implementing a screening protocol, clinicians can practice screening with friends and colleagues, and identify IPV resources available locally and nationally for both clinicians and women. Through all of this, therapeutic and non-judgmental communication is key to creating an optimal environment where women are empowered to share their experiences and get help.

\section{Acknowledgments}

J. Draughon was supported by T32NR007081 from the National Institute of Nursing Research, National Institutes of Health.

\section{References}

1. BreidingMJ, , BasileKC, , SmithSG, , BlackMC, , MahendraR. Intimate Partner Violence Surveillance Uniform Definitions and Recommended Data Elements, Version 2.0Atlanta, GA: National Center for Injury Prevention and Control, Centers for Disease Control and Prevention; 2015http://www.cdc.gov/violenceprevention/pdf/intimatepartnerviolence.pdf [Accessed July 29, 2015]

2. World Health OrganizationGlobal and Regional Estimates of Violence against Women: Prevalence and Health Effects of Intimate Partner Violence and Non-Partner Sexual ViolenceGeneva: World Health Organization; 2013http://apps.who.int/iris/bitstream/ 10665/85239/1/9789241564625_eng.pdf?ua=1 [Accessed October 24, 2015]

3. Silverman JG, Decker MR, Reed E, Raj A. Intimate partner violence victimization prior to and during pregnancy among women residing in 26 U.S. states: associations with maternal and neonatal health. Am J Obstet Gynecol. 2006; 195:140-148. [PubMed: 16813751]

4. Taillieu TL, Brownridge DA. Violence against pregnant women: prevalence, patterns, risk factors, theories, and directions for future research. Aggress Violent Behav. 2010; 15(1):14-35. 
5. Nelson HD, Bougatsos C, Blazina I. Screening women for intimate partner violence: a systematic review to update the 2004 U.S. Preventive Services Task Force recommendation. Ann Intern Med. 2012; 156(11):796-808. [PubMed: 22565034]

6. American College of Nurse-MidwivesPosition Statement: Violence Against WomenSilver Spring, MD; American College of Nurse-Midwives; 2013http:/www.midwife.org/ACNM/files/ ACNMLibraryData/UPLOADFILENAME/000000000091/Violence-Against-Women-Sept-2013.pdf [Accessed July 6, 2015]

7. Todahl J, Walters E. Universal screening for intimate partner violence: a systematic review. J Marital Fam Ther. 2011; 37(3):355-369. [PubMed: 21745237]

8. Sprague S, Madden K, Simunovic N, et al. Barriers to screening for intimate partner violence. Women Health. 2012; 52(6):587-605. [PubMed: 22860705]

9. O'Reilly R, Beale B, Gillies D. Screening and intervention for domestic violence during pregnancy care: a systematic review. Trauma Violence Abuse. 2010; 11(4):190-201. [PubMed: 20688785]

10. American College of Obstetricians and Gynecologists. ACOG Committee opinion no. 554: reproductive and sexual coericon. Obstet Gynecol. 2013; 121(2 Pt 1):411-415. [PubMed: 23344307]

11. Ghandour RM, Campbell JC, Lloyd J. Screening and counseling for intimate partner violence: a vision for the future. J Womens Health. 2015; 24(1):57-61.

12. Iverson KM, Huang K, Wells SY, Wright JD, Gerber MR, Wiltsey-Stirman S. Women veterans' preferences for intimate partner violence screening and response procedures within the Veterans Health Administration. Res Nurs Health. 2014; 37(4):302-311. [PubMed: 24990824]

13. Laughon K, Renker P, Glass N, Parker B. Revision of the abuse assessment screen to address nonlethal strangulation. J Obstet Gynecol Neonatal Nurs. 2008; 37(4):502-507.

14. World Health OrganizationResponding to Intimate Partner Violence and Sexual Violence against Women: WHO Clinical and Policy GuidelinesGeneva: World Health Organization; 2013http:// apps.who.int/iris/bitstream/10665/85240/1/9789241548595_eng.pdf [Accessed October 24, 2015]

15. Bogovic A, Mihanovic M, Jokic-Begic N, Svagelj A. Personal space of male war veterans with posttraumatic stress disorder. Environ Behav. 2013; 46(8):929-945.

16. Morse DS, Lafleur R, Fogarty CT, Mittal M, Cerulli C. "They told me to leave": how health care providers address intimate partner violence. J Am Board Fam Med. 2012; 25(3):333-342. [PubMed: 22570397]

17. Sutherland MA, Fontenot HB, Fantasia HC. Beyond assessment: examining providers' responses to disclosures of violence. J Am Assoc Nurse Pract. 2014; 26(10):587-573.

18. Campbell JC, Webster DW, Glass NE. The danger assessment: validation of a lethality risk assessment instrument for intimate partner femicide. J Interpers Violence. 2009; 24(4):653-674. [PubMed: 18667689]

19. Sohal H, Eldridge S, Feder G. The sensitivity and specificity of four questions (HARK) to identify intimate partner violence: a diagnostic accuracy study in general practice. BMC Fam Pract. 2007; 8:49.doi: 10.1186/1471-2296-8-49 [PubMed: 17727730]

20. Feldhaus KM, Koziol-McLain J, Amsbury HL, et al. Accuracy of 3 brief screening questions for detecting partner violence in the Emergency Department. J Am Med Assoc. 1997; 277(17):13571361.

21. Paranjape A, Liebschutz J. STaT: a three-question screen for intimate partner violence. J Womens Health (Larchmt). 2003; 12(3):233-239. [PubMed: 12804354] 
Table 1

Sample Script for Routine IPV Screening with Use of the Abuse Assessment Screen

\begin{tabular}{|c|c|}
\hline Part of Script & Example Language \\
\hline Normalizing statement & $\begin{array}{l}\text { Next I am going to ask you questions about intimate partner violence. These are questions we ask all of } \\
\text { our patients. }\end{array}$ \\
\hline Mandatory Reporting(if applicable) & $\begin{array}{l}\text { Before I ask you these questions, I do need to tell you that I am a mandated reporter of intimate partner } \\
\text { violence. What that means, is that if you answer yes to any of the following questions, I will have to file } \\
\text { a report with the police on your behalf. }\end{array}$ \\
\hline Normalizing statement & We know that all couples argue. When you and your partner argue... \\
\hline \multirow[t]{4}{*}{ Abuse Assessment Screen (AAS) ${ }^{a}$} & $\begin{array}{l}\text { (1) Within the last year, have you been pushed, shoved, slapped, hit, kicked, choked or otherwise } \\
\text { physically hurt by your partner or ex-partner? }\end{array}$ \\
\hline & $\begin{array}{l}\text { (2) Since you've been pregnant, have you been hit, slapped, kicked, or otherwise physically hut by } \\
\text { someone? }\end{array}$ \\
\hline & (3) Within the last year, has anyone forced you to have sexual activities that you did not want? \\
\hline & (4) Are you afraid of anyone? \\
\hline Negative Screen: & $\begin{array}{l}\text { Thank you for answering. We like to make sure women know this is a safe place to discuss intimate } \\
\text { relationships. }\end{array}$ \\
\hline Positive Screen: & Thank you for sharing with me, I know it can be difficult to talk about these topics. \\
\hline Additional empowering statements & $\begin{array}{l}\text { I believe you. } \\
\text { This was not your fault. } \\
\text { No one deserves to be treated this way. } \\
\text { There is help. }\end{array}$ \\
\hline
\end{tabular}

a A "yes" to any question of the AAS constitutes a positive screen for IPV.

AAS questions reprinted with permission from Laughon et al. 13 
Table 2

Select Intimate Partner Violence Screening Tools ${ }^{a}$

\begin{tabular}{|c|c|c|c|}
\hline Authors & Screening Tool & Validity & Pros/Cons \\
\hline $\begin{array}{l}\text { Laughon et al, } \\
2008^{13}\end{array}$ & $\begin{array}{l}\text { Abuse Assessment } \\
\text { Screen (AAS) }\end{array}$ & $\begin{array}{l}\text { Sensitivity: } 32-93 \% \\
\text { Specificity: } 55-99 \% \\
\text { Positive predictive value: } 33 \% \\
\text { Negative predictive value: } 97 \% 5\end{array}$ & $\begin{array}{l}\text { Pro: } 4 \text { questions } \\
\text { Assesses sexual violence } \\
\text { Assesses non-fatal strangulation } \\
\text { Developed for and tested in pregnant women } \\
\text { Con: Inconsistent psychometrics }\end{array}$ \\
\hline $\begin{array}{l}\text { Sohal et al, } \\
2007^{19}\end{array}$ & $\begin{array}{l}\text { Humiliation, Afraid, } \\
\text { Rape, Kick (HARK) }\end{array}$ & $\begin{array}{l}\text { Sensitivity: } 81 \% \\
\text { Specificity: } 95 \% \\
\text { Positive predictive value: } 83 \% \\
\text { Negative predictive value: } 94 \%\end{array}$ & $\begin{array}{l}\text { Pro: } 4 \text { questions } \\
\text { Assesses sexual violence } \\
\text { High sensitivity and specificity }{ }^{5} \\
\text { Con: Since it is a relatively newer tool, there is less } \\
\text { research supporting its use. }\end{array}$ \\
\hline $\begin{array}{l}\text { Feldhaus et al, } \\
1997^{20}\end{array}$ & $\begin{array}{l}\text { Partner Violence Screen } \\
\text { (PVS) }\end{array}$ & $\begin{array}{l}\text { Sensitivity: } 64.5-71.4 \% \\
\text { Specificity: } 80.3-84.4 \% \\
\text { Positive predictive value: } 51.3-63.4 \% \\
\text { Negative predictive value: } 87.6-88.7 \%\end{array}$ & $\begin{array}{l}\text { Pro: } 3 \text { questions } \\
\text { Con: Does not assess sexual violence }\end{array}$ \\
\hline $\begin{array}{l}\text { Paranjape et al, } \\
2003^{21}\end{array}$ & $\begin{array}{l}\text { Slapped, Threatened, } \\
\text { and Throw (Things) } \\
\text { (STaT) }\end{array}$ & $\begin{array}{l}\text { Sensitivity: } 64-96 \% \\
\text { Specificity: } 75-100 \%\end{array}$ & $\begin{array}{l}\text { Pro: } 3 \text { questions } \\
\text { High sensitivity and specificity } \\
\text { Con: Does not assess sexual violence } \\
\text { Some articles report } 2 \text { yes responses yields higher } \\
\text { sensitivity and specificity }\end{array}$ \\
\hline
\end{tabular}

${ }^{a}$ For all tools, a single "yes" response constitutes a positive screen for IPV. All tools include questions about physical and psychological violence. 
Table 3

\section{Sample Responses for a Positive IPV Screen}

\begin{tabular}{|l|l|}
\hline Part of Response & Example Language \\
\hline $\begin{array}{l}\text { Ask the woman if she wants } \\
\text { assistance at this time }\end{array}$ & $\begin{array}{l}\text { Thank you for answering. I know it can be hard to discuss this topic. Would you like me to help you with } \\
\text { this? }\end{array}$ \\
\hline Offer assistance and choices & $\begin{array}{l}\text { (1) May I share some resources with you?(2) I'd be happy to talk with you about this now, or if you prefer } \\
\text { we can make a follow-up appointment.(3) Would you like me to help you make a call to a hotline that helps } \\
\text { women in this situation? If you would prefer to speak privately, I can set you up with a phone in a private } \\
\text { room.(4) There are several local community resources for women in similar situations; if you would like, I } \\
\text { can help you make an appointment, or we can call one of them together. }\end{array}$ \\
\hline $\begin{array}{l}\text { Encourage safety planning } \\
\text { without coercion }\end{array}$ & $\begin{array}{l}\text { I would like to help you to remain safe while in your relationship. May I share some safety planning } \\
\text { techniques with you? }\end{array}$ \\
\hline $\begin{array}{l}\text { Communicate that resources and } \\
\text { help are available }\end{array}$ & $\begin{array}{l}\text { It's okay if you don't want help now. If you change your mind and want assistance or resources in the } \\
\text { future, I are here and I am happy to help. You are not alone. }\end{array}$ \\
\hline
\end{tabular}


Table 4

Web and National Resources for Screening and Responding to IPV

\begin{tabular}{|l|l|l|}
\hline Name of Resource & Where to Access & Description \\
\hline Danger Assessment & https://www.dangerassessment.org/ & $\begin{array}{l}\text { Training on use of the danger assessment to assess } \\
\text { risk for homicide. }\end{array}$ \\
\hline Futures Without Violence & http://www.futureswithoutviolence.org/ & $\begin{array}{l}\text { Training webinars, resources for clinicians, links to } \\
\text { screening resources. }\end{array}$ \\
\hline National Domestic Violence Hotline & $1-800-799-$ SAFE & $\begin{array}{l}\text { 24-7 hotline for crisis intervention and safety } \\
\text { planning. }\end{array}$ \\
\hline $\begin{array}{l}\text { National Network to End Domestic } \\
\text { Violence }\end{array}$ & http://nnedv.org/ & $\begin{array}{l}\text { Links to state coalitions, safety planning guides, and } \\
\text { get help resources for victims. }\end{array}$ \\
\hline $\begin{array}{l}\text { National Sexual Assault Hotline } \\
\text { Nursing Network on Violence Against } \\
\text { Women }\end{array}$ & $1-800-656-H O P E$ & $\begin{array}{l}\text { Confidential phone support and connection to local } \\
\text { resources. }\end{array}$ \\
\hline $\begin{array}{l}\text { National Online Resource Center on } \\
\text { Violence Against Women }\end{array}$ & http://www.vawnet.org/ & Links to tools for abuse assessment. \\
\hline
\end{tabular}

